\title{
Dual-signal Nanoswitchs for In Situ Intracellular Imaging of non-coding RNA
}

\author{
Xiang-Ling Li, Jin Wang, Jing-Juan Xu*, Hong-Yuan Chen \\ State Key Laboratory of Analytical Chemistry for Life Science and Collaborative Innovation Center of \\ Chemistry for Life Sciences, School of Chemistry and Chemical Engineering, Nanjing University, \\ Nanjing 210023, China \\ Corresponding author's e-mail: xujj@nju.edu.cn
}

\begin{abstract}
:
With the fast development of imaging technology, nanomaterials owned excellent properties have been widely applied in the fields of imaging analysis. However, some shortcomings of imaging technology and nanomaterials, including low stability and sensitivity of probes, the "false positive" results, light bleaching and cell photo damage during in imaging analysis, still exist in the present analytical methods. To address these problems, we designed a novel type of optical nanoprobes owning bifunctional imaging properties, providing insight on fundamental understanding of the signal generation mechanism and transformation mechanism. The novel probes can be further combined with dark-field and laser confocal scanning microscope imaging technique, for establishing a dualimaging analysis strategy for intracellular non-coding RNA. Besides, we plan to extend the application scope of this method, study the intracellular multiple analytes simultaneous and dynamical imaging analysis in real-time, meanwhile imaging at molecule level, thereby provide powerful techniques for biochemical analysis.
\end{abstract}

Key words: plasmonic nanoparticle, DNA scaffolds, plasmon coupling, dark-field imaging, fluorescence imaging.

Noble metal nanoparticles, which have localized surface plasmon resonance (LSPR) in the visible range and do not blink or bleach, have been widely used in biology, chemistry and photonics analysis ${ }^{[1-3]}$. Additionally, DNA scaffolds have been emerged as novel materials for the creation of precisely selfassembled nanostructures due to its programmability and biocompatibility ${ }^{[4-6]}$. Therefore, integrating DNA programmability with nanoparticle functionality in single nanoprobe, can take advantages of both to identify intracellular biomolecule more accurately and sensitively. Herein, we demonstrated newly designed nanoswitchs by the combination of the DNA scaffolds with plasmonic nanoparticles for fluorescence/darkfield dual-modality nanoprobe imaging, Fig. 1. Specifically, asymmetric modified gold nanoparticles (AuNPs) integrated with DNA tetrahedron nanostructures for constructing dual-signal nanoswitchs. When the target, a complementary strand was introduced to the dual properties nanoswitch, more stable hybridization between target and fluorophore labeled oligonucleotide induced the transformation of DNA nanostructures and the recovery of fluorescence signal, achieving fluorescence Imaging. Meanwhile, the transformation of DNA nanostructure led to the formation of an AuNP dimer with significant plasmon resonance coupling effect, resulting in a noticeable scattering signal (Fig.2).

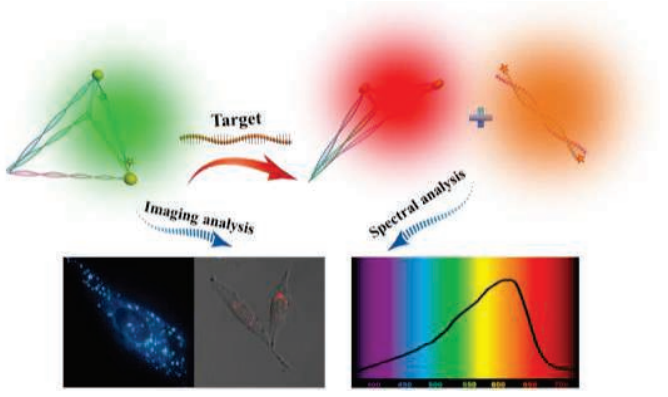

Fig. 1. Schematic representation of single noncoding RNA analysis via dual-signal nanoswitchs. 

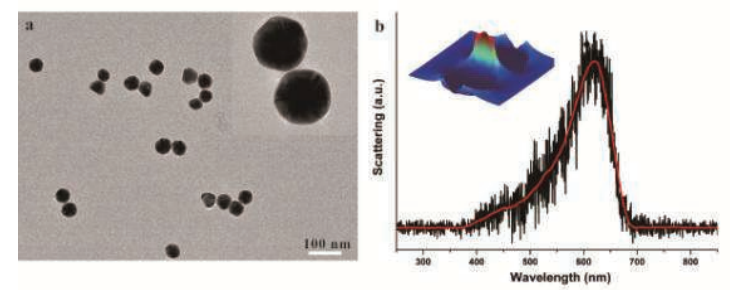

Fig. 2. TEM characterization (a) and scattering spectrum of the nanoswtichs (b) after adding the target molecule. The inset in a is HRTEM image of a probe, the inset in $b$ is Finite-difference time-domain (FDTD) simulation of plasmonic properties for a nanoswtich.

\section{References}

[1] L. Y. T. Chou, F. Song, W. C. W. Chan, Engineering the Structure and Properties of DNANanoparticle Superstructures Using Polyvalent Counterions, Journal of the American Chemical Society 138, 4565-4572 (2016); doi: 10.1021/jacs.6b00751.

[2] R. Elghanian, J. J. Storhoff, R. C. Mucic, R. L. Letsinger, C. A. Mirkin, Selective colorimetric detection of polynucleotides based on the distance-dependent optical properties of gold nanoparticles, Science 277, 1078-1081 (1997); doi:10.1126/science.277.5329.1078.

[3] S. Lal, S. Link, N. J. Halas, Nano-optics from sensing to waveguiding, Nature Photonics 1, 641648 (2007); doi:10.1038/nphoton.2007.223.

[4] D. Bhatia, S. Arumugam, M. Nasilowski, H. Joshi, C. Wunder, V. Chambon, V. Prakash, C. Grazon, B. Nadal, P. K. Maiti, L. Johannes, B. Dubertret, Y. Krishnan, Quantum dot-loaded monofunctionalized DNA icosahedra for singleparticle tracking of endocytic pathways, Nature Nanotechnology. 11, 1112-1119 (2016); doi: 10.1038/nnano.2016.150.

[5] O. I. Wilner, Y. Weizmann, R. Gill, O. Lioubashevski, R. Freeman, I. Willner, Enzyme cascades activated on topologically programmed DNA scaffolds, Nature Nanotechnology 4, 249254 (2009); doi:10.1038/nnano.2009.50.

[6] W. Liu, J. Halverson, Y. Tian, A. V. Tkachenko, O. Gang, Self-organized architectures from assorted DNA-framed nanoparticles, Nature Chemistry 8, 867-873 (2016); doi:10.1038/nchem.2540. 